\title{
A Systematic Analysis of Drosophila Regulatory Peptide Expression in Enteroendocrine Cells
}

\author{
Ji Chen, Seol-min Kim, and Jae Young Kwon*
}

\begin{abstract}
The digestive system is gaining interest as a major regulator of various functions including immune defense, nutrient accumulation, and regulation of feeding behavior, aside from its conventional function as a digestive organ. The Drosophila midgut epithelium is completely renewed every 1-2 weeks due to differentiation of pluripotent intestinal stem cells in the midgut. Intestinal stem cells constantly divide and differentiate into enterocytes that secrete digestive enzymes and absorb nutrients, or enteroendocrine cells that secrete regulatory peptides. Regulatory peptides have important roles in development and metabolism, but study has mainly focused on expression and functions in the nervous system, and not much is known about the roles in endocrine functions of enteroendocrine cells. We systemically examined the expression of 45 regulatory peptide genes in the Drosophila midgut, and verified that at least 10 genes are expressed in the midgut enteroendocrine cells through RT-PCR, in situ hybridization, antisera, and 25 regulatory peptide-GAL transgenes. The Drosophila midgut is highly compartmentalized, and individual peptides in enteroendocrine cells were observed to express in specific regions of the midgut. We also confirmed that some peptides expressed in the same region of the midgut are expressed in mutually exclusive enteroendocrine cells. These results indicate that the midgut enteroendocrine cells are functionally differentiated into different subgroups. Through this study, we have established a basis to study regulatory peptide functions in enteroendocrine cells as well as the complex organization of enteroendocrine cells in the Drosophila midgut.
\end{abstract}

\section{INTRODUCTION}

Regulatory peptides are known to have major roles in animal physiology, such as regulation of metabolism. Most previous studies focused on the functional roles of regulatory peptides in

Department of Biological Sciences, Sungkyunkwan University, Suwon 16419, Korea

${ }^{*}$ Correspondence: jykwon@ @skku.edu

Received 25 January, 2016; revised 16 February, 2016; accepted 18 February, 2016; published online 30 March, 2016

Keywords: Drosophila melanogaster, enteroendocrine cells, regulatory peptides the central nervous system. In recent years, the roles of regulatory peptides in enteroendocrine cells have been gaining interest (Wegener and Veenstra, 2015).

The enteroendocrine system is the primary sensor of ingested food in vertebrates, and is responsible for secreting various gastrointestinal hormones. These peptide hormones include cholecystokinin (CCK), glucagon-like peptide 1 (GLP1), glucose-dependent insulinotropic peptide (GIP), somatostatin, ghrelin, peptide YY (PYY), and serotonin (5-hydroxytryptamine [5-HT]). Peptide hormones work together to regulate physiological responses such as gastrointestinal motility and secretion, glucose homeostasis, and appetite (Psichas et al., 2015). Enteroendocrine cells dispersed throughout the intestinal tract produce and secrete enteroendocrine peptides hormones, and were found to express a broad repertoire of peptide hormone precursors in studies of transgenic enteroendocrine reporter mice (Engelstoft et al., 2013). Despite the vast number of enteroendocrine peptides predicted to exist in vertebrates (Psichas et al., 2015), the functions of most remain to be elucidated.

The Drosophila melanogaster genome contains at least 45 neuropeptide genes, which encode much larger numbers of mature neuropeptides (Hansen et al., 2011; Nassel and Winther, 2010; Veenstra and Ida, 2014). In this study, we examine the expression of neuropeptide genes in enteroendocrine cells in the midgut, and thus we use the terms regulatory peptides or enteroendocrine peptides to emphasize expression in the intestine. Study of the expression and function of enteroendocrine peptides in the fly system can provide useful insight, since some peptide hormones have sequence and functional homology to their vertebrate counterparts (Brogiolo et al., 2001; Brown et al., 1999; Lee and Park, 2004; Lee et al., 2004; 2008; Melcher and Pankratz, 2005; Park et al., 2014; Wu et al., 2003).

The Drosophila digestive tract can be largely divided into the foregut, midgut, and hindgut, and enteroendocrine cells are known to be only present in the midgut (Veenstra et al., 2008). Drosophila melanogaster provides a genetically tractable model system to study the intestine, and has gained interest in recent years following the discovery of intestinal stem cells in the midgut (Micchelli and Perrimon, 2006; Ohlstein and Spradling, 2006). Drosophila enteroendocrine cells differentiate from intestinal stem cells, and are dispersed over the entire midgut (Beehler-Evans and Micchelli, 2015; Zeng and Hou, 2015). Regulatory peptides expressed in the enteroendocrine cells have been suggested to have roles in digestive enzyme secretion in response to intestinal nutrient contents, regulation of gut motility and food intake, as well as involvement in intestinal stem cell proliferation and differentiation in a paracrine manner 
(Beehler-Evans and Micchelli, 2015; Scopelliti et al., 2014; Wang et al., 2015; Wegener and Veenstra, 2015). Previous studies on the Drosophila enteroendocrine peptides reported on the expression of regulatory peptides in the midgut through peptidomic LC/MS analysis (Reiher et al., 2011), staining with peptide antisera (Veenstra et al., 2008), or in situ hybridization to individual peptide genes (Brown et al., 1999; Price et al., 2002; Siviter et al., 2000; Williamson et al., 2001), mainly focusing on the expression of individual regulatory peptide genes, or the use of a single methodology to examine regulatory peptide expression.

Here, using RT-PCR, in situ hybridization, and 25 regulatory peptide-GAL transgenes, we conduct the most comprehensive analysis of Drosophila midgut enteroendocrine peptide expression to date. In addition, by using antisera and GAL4 drivers together, we complete a comprehensive analysis of enteroendocrine cell subpopulations that express specific combinations of enteroendocrine peptides. Thus, we establish a basic point of reference for further studies on the physiological roles of enteroendocrine peptides in the midgut as well as enteroendocrine cell differentiation.

\section{MATERIALS AND METHODS}

\section{Drosophila stocks and transgenic flies}

Flies were grown on standard cornmeal/agar culture medium at an average culture temperature of $23^{\circ} \mathrm{C}$. For all experiments, no obvious gender differences were observed between male and female flies. Data for $7 \pm 2$ day old females is presented for all experiments. Flies were synchronized by placing 10 each of freshly eclosed male and female flies in fresh food vials and flipping the flies to fresh food every 2-3 days. $w^{1118}$ flies were used for total mRNA extraction and in situ hydridization experiments. Immunostaining was performed on transgenic flies containing both a regulatory peptide-GAL4 transgene and UAS$m C D 8-G F P$ as a GFP reporter (Lee and Luo, 1999). A total of 25 regulatory peptide-GAL4 drivers were used, with details on 23 described elsewhere (Min et al., 2016), and AstA-GAL4 (Hergarden et al., 2012) and Dh31-GAL4 (Bloomington stock \#46389).

\section{$R T-P C R$ amplification of regulatory peptide gene transcripts in the midgut}

RT-PCR was performed for 37 regulatory peptide genes (Supplementary Fig. S1 and Supplementary Table S1). Since primers were designed in exon sequences flanking an intron to distinguish between genomic DNA and CDNA amplification, 7 genes that consist of a single exon (MIP, amnesiac, FMRFamide, Leucokinin, Dilp1, Pdf, and Drosulfakinin) and the orcokinin gene that had not been defined at the start of our study were not included in the analysis. With the exception of Gpb5, Nplp1, and ITP, primer sets were designed to amplify and detect all predicted alternative transcripts. Three alternative transcripts are predicted for Gpb5, and we used a primer set that can detect one transcript that contains an intron. For Np/p1, we used a primer set that can detect two transcripts out of the predicted three. Five alternative transcripts are predicted for ITP in Flybase, and we used two primer sets, ITP(1) and ITP(2), that amplify the ITP-RC form or the ITP-RD, RF, and RG form, respectively. This resulted in a total of 38 primer pairs used to amplify the 37 regulatory peptide genes (Supplementary Table S2). Over 80 flies were dissected to collect intestines, and gut total RNA was extracted using the RNAiso Plus kit (Takara). The RNeasy kit (Qiagen, catalog no. 74104) was used to clean the extracted total RNA. Reverse transcription was performed using the PrimeScript $1^{\text {st }}$ strand cDNA Synthesis kit (Takara), and PCR was performed for 35 cycles using genomic DNA or the synthesized cDNA as templates.

\section{High-throughput data analysis}

The RT-PCR results were compared with high-throughput expression data including FlyAtlas Anatomy Microarray Data and modENCODE Anatomy RNA-Seq data (Supplementary Table S1). Adult midgut expression level data was extracted from FlyAtlas Anatomy Microarray Data, and digestive system and 4day adult RNA-Seq data was extracted from the modENCODE Anatomy RNA-Seq database (http://flybase.org/; scores in Table S1 were last updated on January 11th, 2016). Although not quantifiable, 13 of the 22 regulatory peptide genes amplified by RT-PCR showed clear and strong bands, and 9 showed relatively weak bands (Supplementary Fig. S1 and Supplementary Table S1). These results correlated well with the highthroughput expression data. Genes that showed strong RTPCR bands had microarray scores ranging from 40.9 (ITP) to 1441.9 (AstC), and genes with weak RT-PCR bands had microarray scores below 18.7, with the exception of Dh31 that had a score of 113.6. The 15 genes that were not amplified by RT-PCR all had microarray scores below 12. Similarly, the 15 genes that were not amplified by RT-PCR had RNA-Seq levels of 0 , with the exception of the sex peptide gene which was a level 5 . For the 8 genes that we did not perform RT-PCR, microarray scores were below 1.8 and RNA-Seq levels were 0 , with the exception of MIP (microarray, 39.6; RNA-Seq, 2) and orcokinin (microarray, 36.3; RNA-Seq, 14). To select genes to verify by in situ hybridization, we first selected the 13 genes that showed a strong signal in RT-PCR experiments. Next, we selected $A K H, D h 31, P T T H$, and SNPF from the 9 genes with weak RT-PCR bands, excluding the genes CCAP, ETH, EH, GPB5, and Dilp6 that had microarray scores below 4.5 and RNA-Seq levels equal to or lower than 2. MIP was added to the list, because of previous results showing expression in the intestine (Veenstra et al., 2008) and high microarray scores. Nplp1 was also added to the list, to verify the expression of all Nplp genes, Nplp1-4. This resulted in a total of 19 genes that were subsequently examined by in situ hybridization.

\section{In situ hybridization}

In situ hybridization was carried out basically following a previously described protocol (Chen et al., 2015). Intestinal tissues from 5-9 day-old adults were dissected in phosphate-buffered saline with $0.2 \%$ Triton X-100 (PBS-T). Whole abdomens with incisions were fixed and subjected to subsequent staining steps and the intestine was dissected out for mounting. Samples were fixed in $4 \%$ paraformaldehyde in PBS-T for $2-4 \mathrm{~h}$ at room temperature, and washed with PBS-T. Proteinase $\mathrm{K}(50 \mu \mathrm{g} / \mathrm{ml}$ in PBS-T) treatment was performed for $10 \mathrm{~min}$, and the reaction stopped by adding glycine-PBS-T (2 mg glycine/ml PBS-T). After washing with PBS-T, tissues were postfixed in $4 \%$ paraformaldehyde in PBS-T for $1 \mathrm{~h}$ and washed with PBS-T only, $1: 1$ hybridization solution ( $50 \%$ formamide, $5 x$ SSC, $0.1 \%$ Tween 20): PBS-T, and hybridization solution only. Following prehybridization in hybridization solution for $10-20$ min at $48^{\circ} \mathrm{C}$, dissected tissues were incubated with ssDNA probes in hybridization solution for at least $20 \mathrm{~h}$ at $48^{\circ} \mathrm{C}$. Digoxygenin (DIG)labeled ssDNA probes were prepared using the PCR-DIG Probe Synthesis kit (Roche). The primer sets used to construct in situ probes are listed in Supplementary Table S3. Probes were mixed into hybridization solution $(10 \mu \mathrm{l}$ of probe in $90 \mu \mathrm{l}$ of 
hybridization solution) and boiled for 40-45 min just before hybridization. After hybridization, the dissected tissues were washed with hybridization solution for $2-4 \mathrm{~h}$ at $48^{\circ} \mathrm{C}$, and briefly washed with hybridization solution only, 1:1 hybridization solution : PBS-T, and PBS-T only. Samples were next blocked with $3 \%$ goat serum in PBS-T for 30 min at room temperature. Alkaline phosphatase-labeled sheep anti-digoxygenin antibody (1:1000, Roche, Germany) was used to amplify the digoxygenin signal overnight at $4^{\circ} \mathrm{C}$. Tissues were subsequently stained with nitro blue tetrazolium and 5-bromo-4-chloro-3-indolylphosphate solution (NBT-BCIP, Roche) diluted 1:50 in alkaline phosphatase buffer (100 mM Tris, $50 \mathrm{mM} \mathrm{MgCl}_{2}, 100 \mathrm{mM} \mathrm{NaCl}$, $0.1 \%$ Tween $20, \mathrm{pH} 9.5)$.

\section{Immunostaining}

For the immunohistochemistry of intestinal cells, whole abdomens were stained and the stained intestines were dissected out for mounting before imaging. A previous immunocytochemistry protocol was followed (Park and Kwon, 2011), with minor modifications. Before dissection, flies were moved to a tube containing a filter paper circle soaked in sucrose solution for $4 \mathrm{~h}$ in order to eliminate cornmeal auto fluorenscence. Dissected abdomens were fixed for at least $2 \mathrm{~h}$ on ice in $4 \%$ paraformaldehyde dissolved in phosphate buffered saline (PBS-T, pH 7.2) containing $0.2 \%$ Triton X-100. After 3 washes of 20 min each in PBS-T, samples were blocked for $2 \mathrm{~h}$ in PBS-T containing $3 \%$ normal goat serum. Abdomens were incubated overnight at $4^{\circ} \mathrm{C}$ with the primary antibody diluted in blocking solution. Washes in PBS-T $(3 \times 20 \mathrm{~min})$ were followed by incubation for over 7 hours with the secondary antibody diluted in blocking solution. Samples were rinsed $3 \times 20$ min in PBS-T and mounted in 50\% glycerol in PBS-T. 4',6-diamidino-2-phenylindole (DAPI, Sigma) was added to the last wash at a final concentration of $0.5 \mu \mathrm{g} / \mathrm{ml}$. Unless otherwise noted, all steps were carried out at room temperature. The primary antibodies used were mouse or rabbit anti-GFP (1:1000) (Molecular Probes); mouse anti-Prospero (1:10) (Developmental Studies Hybridoma Bank at the University of lowa); mouse anti-Drosophila orcokinin B (1:1000) (Veenstra and Ida, 2014); rabbit anti-dNPF (1:1000) (Dr. M. R. Brown, University of Georgia, USA); rabbit antiLeucokinin (1:200) (Chen et al., 1994); rabbit anti-Burs (1:250) (Luan et al., 2006); rabbit anti-AstA, rabbit anti-AstC, rabbit antiDh31 (1:1000) (Dr. J. A. Veenstra, Universite de Bordeaux, France). The anti-dNPF antiserum was preincubated with FMRF peptides $\left(25 \mu \mathrm{g} / \mathrm{ml}\right.$, Sigma P4898) at $4^{\circ} \mathrm{C}$ overnight before use. The secondary antibodies used were goat anti-mouse and goat anti-rabbit IgG conjugated to either Alexa 568 or Alexa 488 (1:1000) (Molecular Probes).

\section{Imaging and microscopy}

The number of Prospero- or GFP-positive enteroendocrine cells for regulatory peptide-GAL4 drivers were estimated on a Leica DM2500 fluorescent microscope. A Zeiss LSM 510, 700 laser-scanning confocal microscope was used to image fluorescent samples, and a Leica DM2500 microscope with a digital camera (Canon EOS 700D) was used to image in situ hybridized samples.

\section{RESULTS AND DISCUSSION}

To analyze the expression of predicted regulatory peptide genes in the Drosophila intestine, we first attempted to amplify transcripts of the regulatory peptide genes by RT-PCR using mRNA isolated from the fly intestine as template (see "Materials and Methods" for details). Using primer sets designed to flank an intron, that can be used to distinguish between amplification of genomic DNA or cDNA (Supplementary Table S2), 37 regulatory peptide genes were tested for expression by RT-PCR, and the transcripts of 22 genes were amplified (Supplementary Fig. S1 and Table S1). Among these, 9 genes (AstA, AstC, Burs, CCHa1, CCHa2, Dh31, Dilp3, NPF, and tachykinin) were previously shown to express in the adult intestine through antisera staining, in situ hybridization, or GAL4-driven expression (Wegener and Veenstra, 2015). These genes rank within the top ten regulatory peptide genes with the highest scores in expression level score data for regulatory peptide gene expression in the midgut extracted from FlyAtlas Anatomy Microarray Data (Supplementary Table S1). The results of high-throughput expression data and our RT-PCR experiments correlated well with previously observed peptide expression, indicating that our RTPCR experiments and the high-throughput expression data provide reliable reflections of expression. Through comparison of the RT-PCR results and high-throughput expression data including FlyAtlas Anatomy Microarray Data and modENCODE Anatomy RNA-Seq data, we excluded 5 genes with markedly low scores in high-throughput expression data (CCAP, ETH, $E H$, GPB5, and Dilp6), and included 2 additional genes, MIP and $N p / p 1$, to result in 19 regulatory peptide genes selected for further in situ hybridization analysis (Supplementary Table S3; see "Materials and Methods" for details).

In situ hybridization showed that 7 regulatory peptide genes including AstA, MIP, AstC, CCHa1, CCHa2, Dh31, and tachykinin express in the midgut (Fig. 1A). AstA, CCHa1, and Dh31 are mainly expressed in the posterior midgut, MIP in the middle midgut and posterior midgut, and $\mathrm{AstC}, \mathrm{CCHa2}$, and tachykinin show expression over the entire length of the midgut (Fig. 1A). All of these genes were expressed in characteristic enteroendocrine cells (Fig. 1B). Burs and NPF were not detected by in situ hybridization, but detected by immunostaining (Fig. 2A). Since NPF in situ hybridization had previously been observed in the adult midgut (Brown et al., 1999), we tested our in situ probe by conducting NPF in situ hybridization and NPF immunostaining in the adult brain and observed very similar expression (Supplementary Fig. S2), suggesting that regulation of expression may exist for NPF in the gut. Similarly for Nplp2, Nplp3, and Nplp4, strong bands were observed by RT-PCR but in situ hybridization did not yield expression in the midgut. This was especially unexpected for Nplp2, which is ranked in the top ten regulatory peptide genes with the highest scores in expression level score data for regulatory peptide gene expression in the midgut extracted from FlyAtlas Anatomy Microarray Data. Under starvation conditions, tachykinin expression in enteroendocrine cells was shown to decrease in order to modulate lipogenesis in enterocytes (Song et al., 2014). Thus, it is likely that the expression of certain enteroendocrine peptides may fluctuate depending on factors such as nutritional state, dietary conditions, or intestinal bacteria composition. Also, certain peptides may express in the midgut muscle, such as Dilp3 (Veenstra et al., 2008), or enteric neurons or malpighian tubules, leading to weak RT-PCR bands when using mRNA from dissected intestines as template.

All peptides whose expression was examined by immunostaining are expressed in similar regions as the in situ results (Fig. 2A) and in characteristic enteroendocrine cells (Fig. 2B), consistent with in situ results. These antibodies were used in subsequent experiments defining different populations of enteroendocrine cells that express different combinations of regulatory peptides (Figs. 4-6). 
A
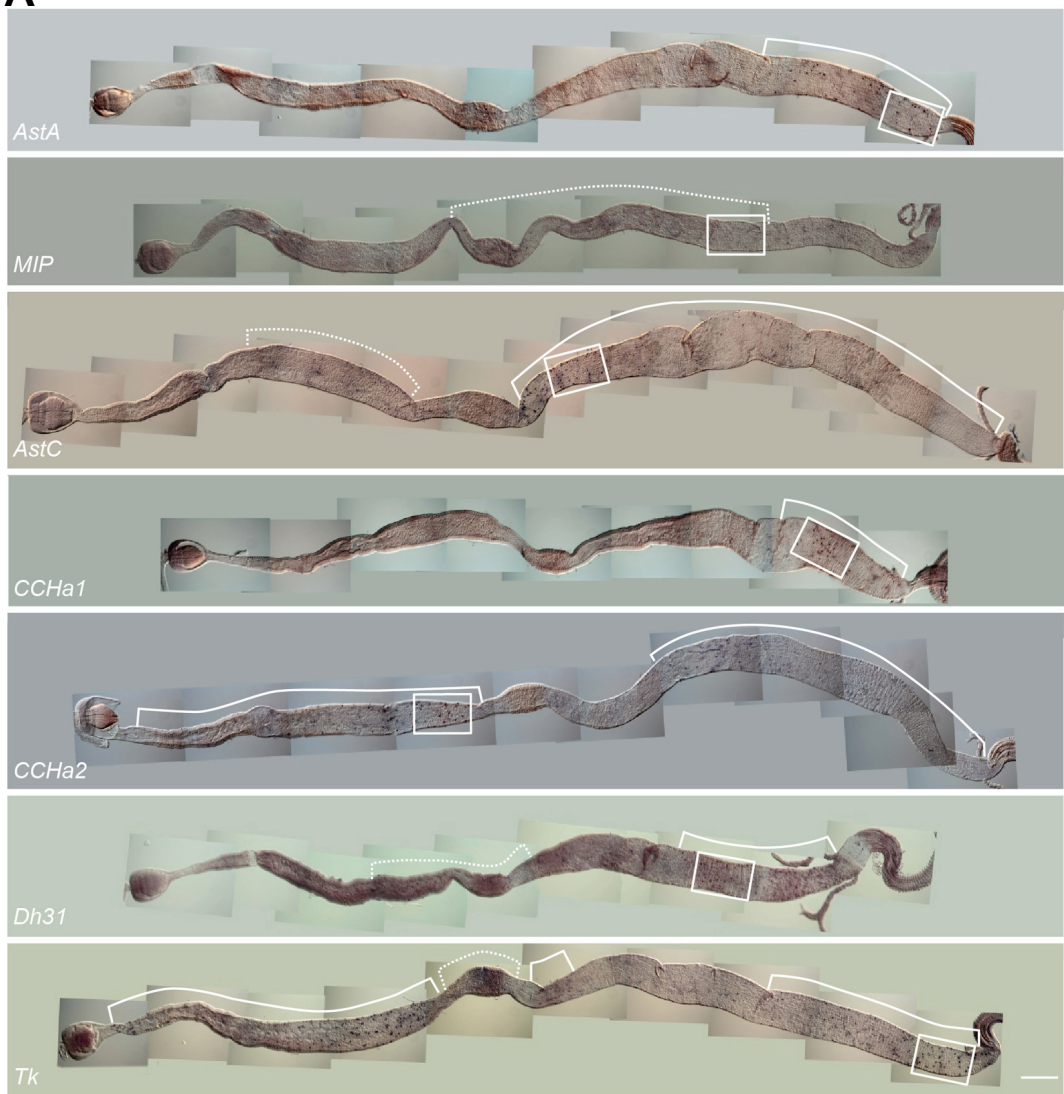

B
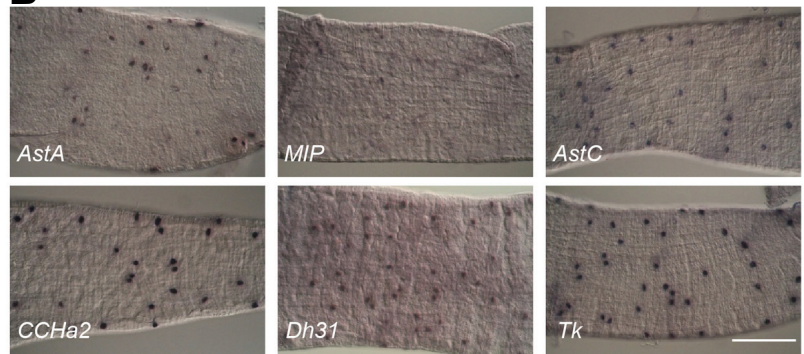

Fig. 1. In situ hybridization of regulatory petide genes in the midgut. (A) Expression patterns of indicated regulatory peptide genes. Solid lines indicate the regions of the midgut that show expression, and the dotted lines indicate regions where the density of expressing cells is relatively low. Anterior is to the left. (B) Magnified images of the boxes in (A). The scale bars indicate $200 \mu \mathrm{m}$.
Next, the GAL4/UAS system was utilized to examine intestinal expression of Drosophila regulatory peptides. Among the 25 regulatory peptide-GAL4 drivers examined (Supplementary Table S1), 6 GAL4 drivers drove GFP reporter expression in the midgut (Fig. $3 A$ ). To verify if the expression driven by GAL4 transgenes represents in vivo peptide expression, we costained with peptide antibodies used in previous studies. Staining with the AstA, AstC, Dh31, and NPF antibodies corresponded well with the expression of each respective GAL4 driver (Fig. 3B). The GFP reporter expression driven by regulatory peptide-GAL4 transgenes shows expression in additional cells compared to the cells marked by immunostaining (Fig. 3B). This is likely due to ectopic expression of the GAL4 transgenes. However, we cannot completely rule out the possibility of expression at a low level undetectable by immunostaining.

As a result of the expression analysis results from in situ hybridization, antibody staining, and regulatory peptide-GAL4 driver expression, a total of 10 regulatory peptide genes were found to express in enteroendocrine cells in the midgut, in specific compartments of the midgut. AstA was observed to express specifically in the posterior midgut in all three experimental methods. Burs, CCHa1, and Dh31 are also mainly expressed in the posterior midgut. NPF and orcokinin are mainly expressed in the anterior and middle midgut. MIP is expressed in the middle midgut and part of the adjacent posterior midgut. AstC, $\mathrm{CCHa} 2$, and tachykinin are expressed broadly over the entire midgut.

To further examine the combinations of regulatory peptide expression that define different populations of enteroendocrine cells in midgut compartments, we used regulatory peptide antibodies and GAL4 drivers to examine co-expression of the peptides. The Drosophila midgut is largely compartmentalized into the anterior, middle, and posterior midgut (Veenstra et al., 2008). Copper cells exist in the middle midgut, with similar cell morphology and acid-secreting functions to parietal cells in the vertebrate stomach (Dubreuil, 2004). The posterior midgut is 
A
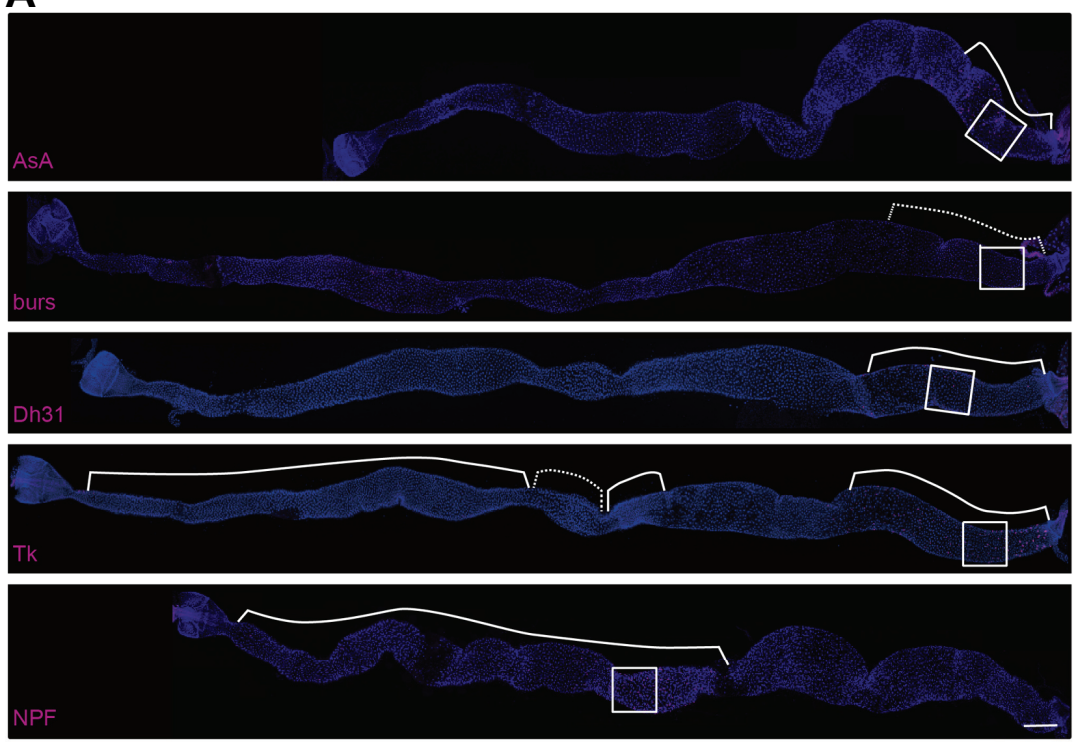

B
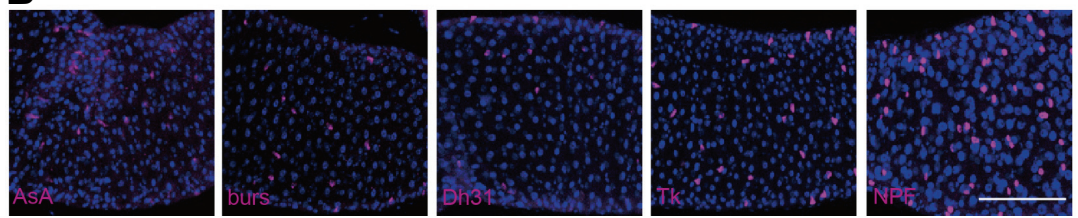

A
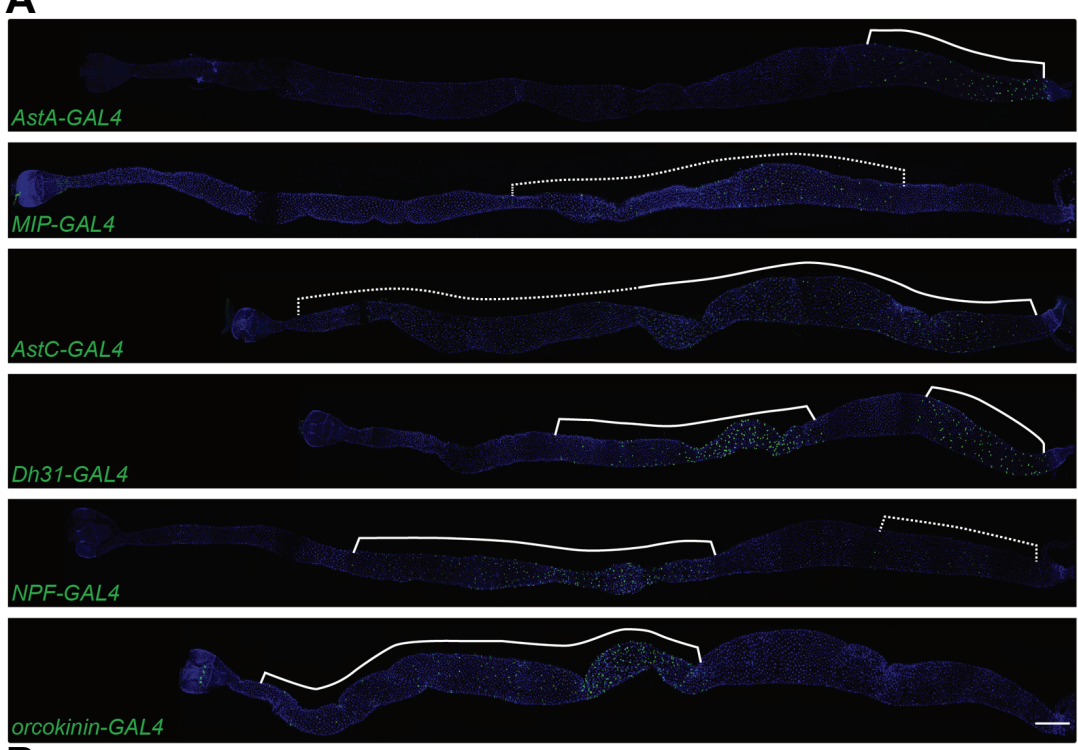

B
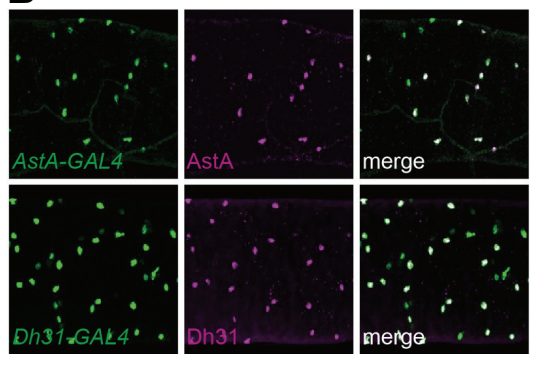

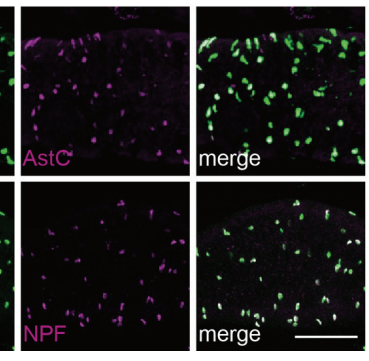

Fig. 2. Immunostaining of regulatory peptides in the midgut. (A) Expression patterns of indicated regulatory peptide genes. Solid lines indicate the regions of the midgut that show expression, and the dotted lines indicate regions where the density of expressing cells is relatively low. Blue staining is due to DAPI staining, and marks nuclei while allowing visualization of the outline of the midgut. (B) Magnified images of the boxes in $(A)$. The scale bars indicate 200 $\mu \mathrm{m}$.

Fig. 3. Regulatory peptide-GAL4 expression in the midgut. (A) Expression patterns of indicated regulatory peptide-GAL4 drivers visualized by GFP reporter expression. Solid lines indicate the regions of the midgut that show expression, and the dotted lines indicate regions where the density of expressing cells is relatively low. Blue shows DAPI staining. (B) AstA-, AstC-, Dh31-, and NPF-GAL4 expression represents the expression of each peptide. Double-color staining of GFP reporter expression driven by regulatory peptideGAL4 (green) and regulatory peptide antibodies (magenta). Photos of AstA and Dh31 show the posterior midgut, and AstC and NPF show the anterior midgut. The scale bars indicate $200 \mu \mathrm{m}$. 
Table 1. Number of cells expressing each regulatory peptide-GAL4 driver in each region of the Drosophila midgut.

\begin{tabular}{lcccc}
\hline & Anterior & Middle & Anterior of posterior & Posterior of posterior \\
\hline Prospero positive cells & $495 \pm 34$ & $239 \pm 25$ & $163 \pm 30$ & $218 \pm 23$ \\
& $(\mathrm{n}=22)$ & $(\mathrm{n}=29)$ & $(\mathrm{n}=27)$ & $(\mathrm{n}=33)$ \\
AstA-GAL4 & & & & $96 \pm 10$ \\
& & & & $(\mathrm{n}=32)$ \\
MIP-GAL4 & & $80 \pm 16$ & $(\mathrm{n}=30)$ & \\
& & $(\mathrm{n}=33)$ & $108 \pm 13$ & $117 \pm 15$ \\
AstC-GAL4 & $128 \pm 13$ & $(\mathrm{n}=15)$ & $(\mathrm{n}=15)$ \\
Dh31-GAL4 & $(\mathrm{n}=29)$ & & $104 \pm 9$ \\
& $(\mathrm{n}=34)$ & $150 \pm 11$ & & $(\mathrm{n}=40)$ \\
NPF-GAL4 & $48 \pm 13$ & $(\mathrm{n}=8)$ & & $61 \pm 7$ \\
& $(\mathrm{n}=8)$ & $122 \pm 15$ & & $(\mathrm{n}=14)$
\end{tabular}

The numbers shown are average \pm SEM.

considered the main site of nutrient absorption (Veenstra et al., 2008). In the anterior midgut (Fig. 4A), AstC and orcokinin are co-expressed in the same enteroendocrine cells, and NPF is expressed in enteroendocrine cells independent of the AstCand orcokinin-expressing enteroendocrine cells (Fig. 4B). Tachykinin is co-expressed with NPF, and Dh31-GAL4-driven GFP reporter expression is expressed in a small number of cells in the anterior midgut, in a subset of the NPF- and tachykinin-expressing enteroendocrine cells (Fig. 4B). In the middle midgut (Fig. 5A), AstC and orcokinin co-express in the same cells, and NPF is expressed in different enteroendocrine cells from those cells (Fig. 5B), similar to the anterior midgut. Dh31 and tachykinin are both co-expressed with NPF (Fig. 5B), also similar to the anterior midgut. MIP-GAL4-expressing cells are a subset of NPF-expressing enteroendocrine cells (Fig. 5B). In the anterior portion of the posterior midgut (Fig. 6A), both AstC and MIP are expressed, with MIP-GAL4-expressing cells a subset of AstC-expressing enteroendocrine cells (Fig. 6B). In the caudal half of the midgut (Fig. 6C), AstC and AstA are coexpressed (Fig. 6D). Dh31 is expressed in completely independent enteroendocrine cells from the AstC- and AstAexpressing cells, and is co-expressed with Burs, tachykinin, and NPF (Fig. 6D).

We quantified the number of enteroendocrine cells expressing individual regulatory peptide-GAL4 drivers in each compartment of the midgut (Fig. 7 and Table 1). Prospero is a marker for enteroendocrine cells in the Drosophila midgut (Micchelli and Perrimon, 2006), with more than 1,100 cells staining for Prospero (Table 1). All regulatory peptide-GAL4-expressing cells were a subset of Prospero-positive cells (Table 1; Supplementary Fig. S3). Enteroendocrine cells in the anterior midgut can be divided into AstC-GAL4-expressing enteroendocrine cells and NPF-GAL4-expressing enteroendocrine cells, with roughly $40 \%$ of enteroendocrine cells expressing none of the GAL4 drivers that were tested (Fig. 7). Enteroendocrine cells in the middle midgut can also be divided into AstC-GAL4expressing enteroendocrine cells and NPF-GAL4-expressing enteroendocrine cells, with orcokinin being co-expressed with AstC, and Dh31, MIP, and tachykinin co-expressed with NPF (Fig. 7). In the anterior half of the middle portion of the posterior midgut, two-thirds of Prospero-positive cells express AstC-, and MIP-GAL4-expressing cells are a subset of these cells (Fig. 7). None of the tested GAL4 drivers showed expression in the

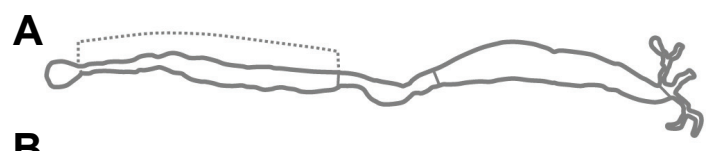

B
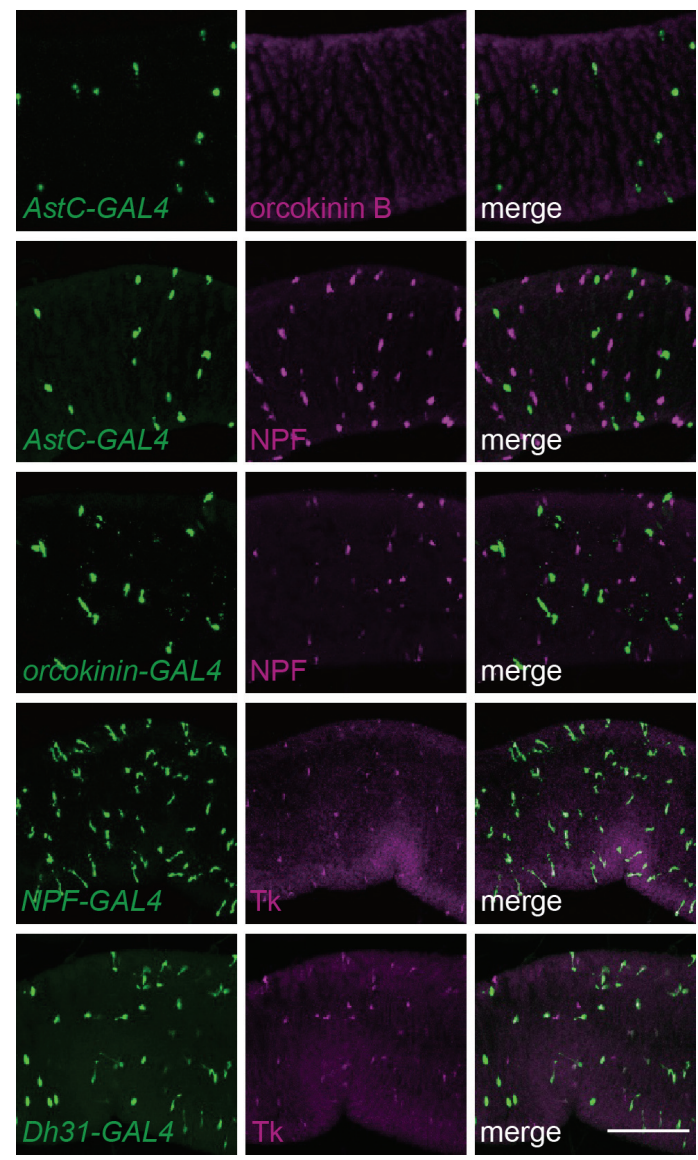

Fig. 4. Regulatory peptides expressed in the anterior midgut. (A) Schematic of the Drosophila midgut, with the dotted line indicating the anterior midgut. (B) Double labeling of indicated regulatory peptide-GAL4-driven GFP reporter expression (green) and antibodies (magenta). The scale bar indicates $200 \mu \mathrm{m}$. 

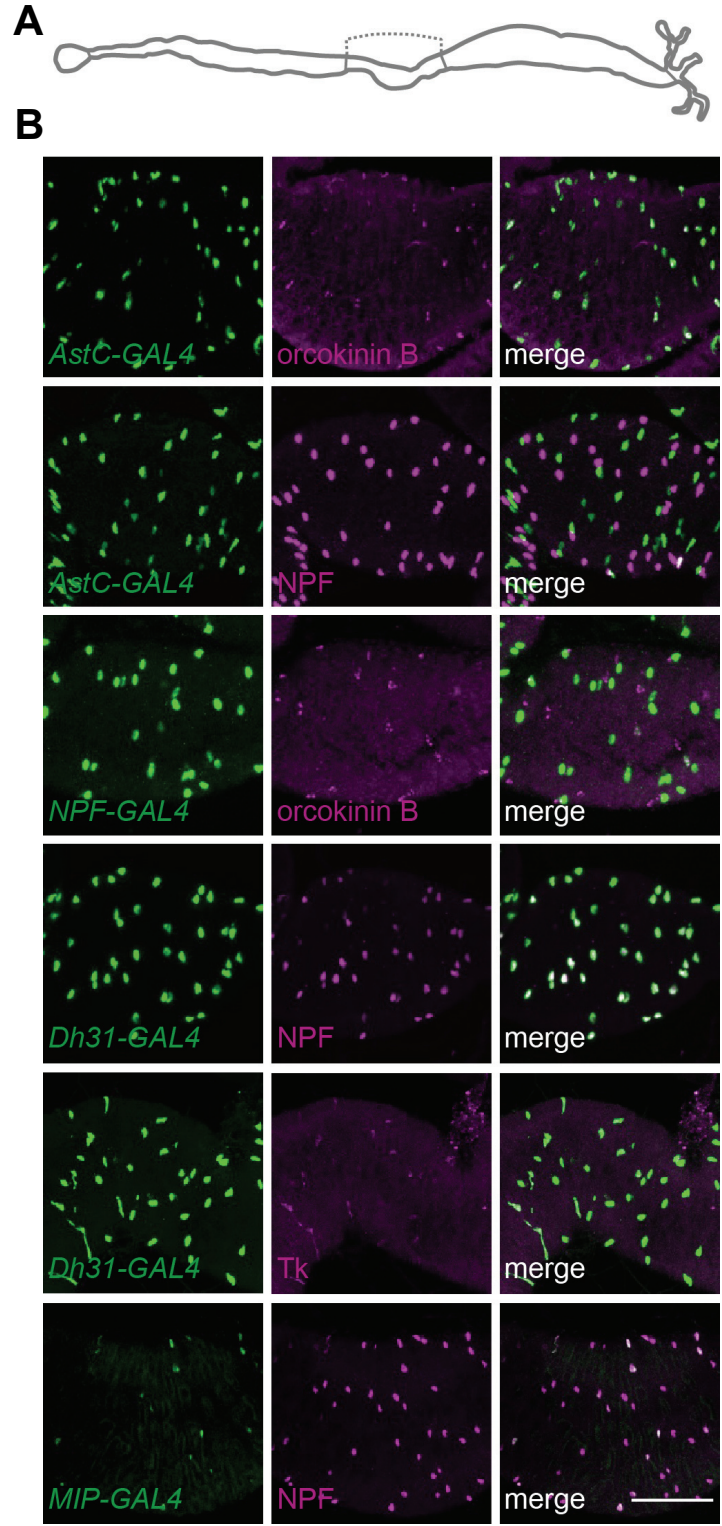

Fig. 5. Regulatory peptides expressed in the middle midgut. (A) Schematic of the Drosophila midgut, with the dotted line indicating the middle midgut. (B) Double labeling of indicated regulatory peptide-GAL4-driven GFP reporter expression (green) and antibodies (magenta). The scale bar indicates $200 \mu \mathrm{m}$.

remaining one-third of Prospoero-positive cells. MIP-GAL4 is unique in that it is expressed exclusively from AstC-GAL4 expressing cells in the middle midgut, but is co-expressed with AstC-GAL4 expressing cells in the posterior midgut. The caudal half of the posterior midgut can be divided into two groups of enteroendocrine cells, with one group co-expressing AstC and AstA, and the other group co-expressing NPF, Dh31, Burs, and tachykinin (Fig. 7).

Overall, Drosophila enteroendocrine cells in the midgut can be largely divided into two populations. One group expresses AstC over the entire midgut, while additionally expressing orcokinin in the anterior and middle midgut, and MIP and AstA in

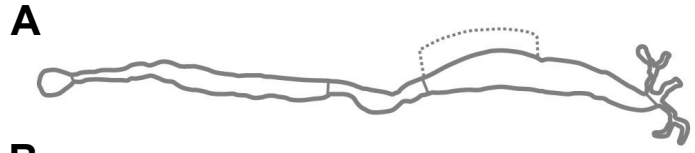

B
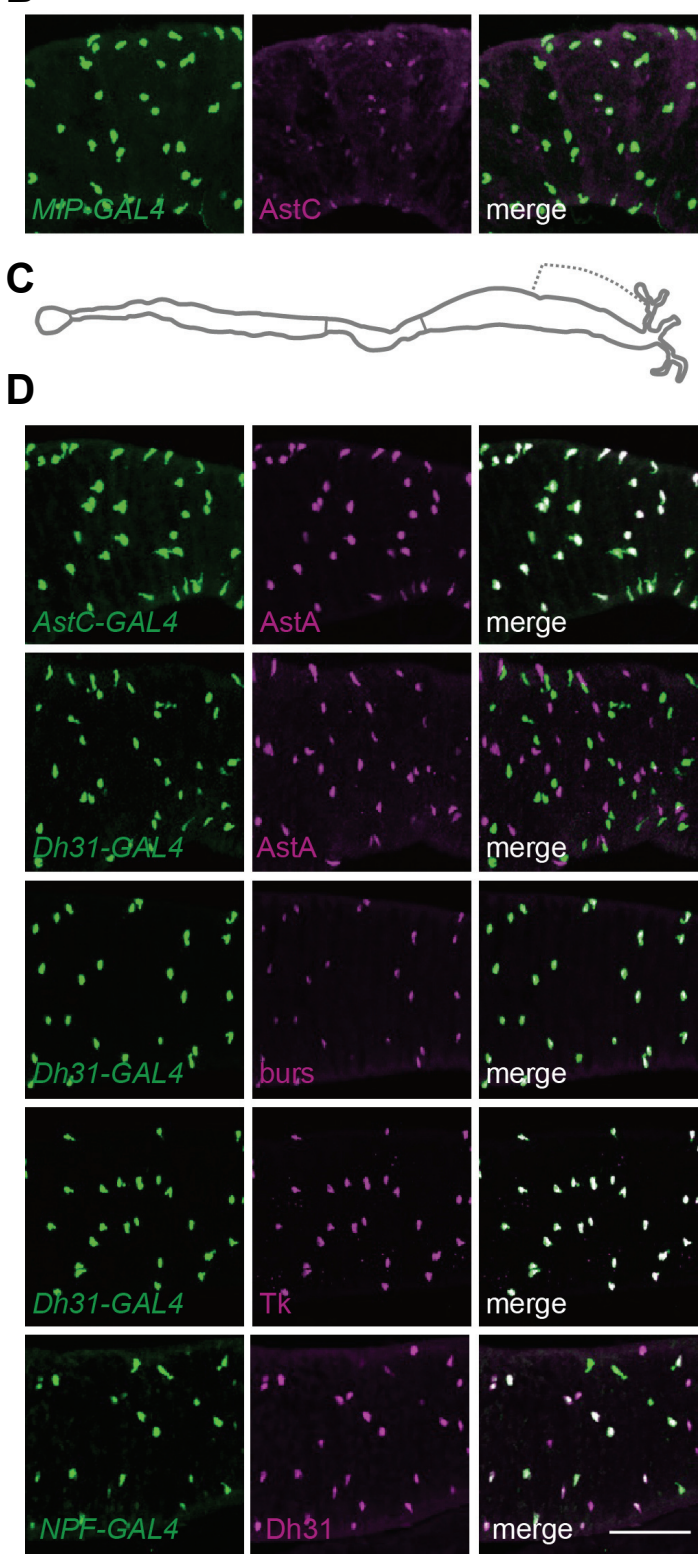

Fig. 6. Regulatory peptides expressed in the posterior midgut. (A) Schematic of the Drosophila midgut, with the dotted line indicating the anterior part of the posterior midgut. (B) Double labeling of MIPGAL4 driven GFP reporter expression (green) and AstC antibody (magenta). (C) Schematic of the Drosophila midgut, with the dotted line indicating the caudal half of the posterior midgut. (D) Double labeling of indicated regulatory peptide-GAL4 driven GFP reporter expression (green) and antibodies (magenta). The scale bar indicates $200 \mu \mathrm{m}$.

the anterior-half and caudal-half of the posterior midgut, respectively. The other group expresses tachykinin over the length of 


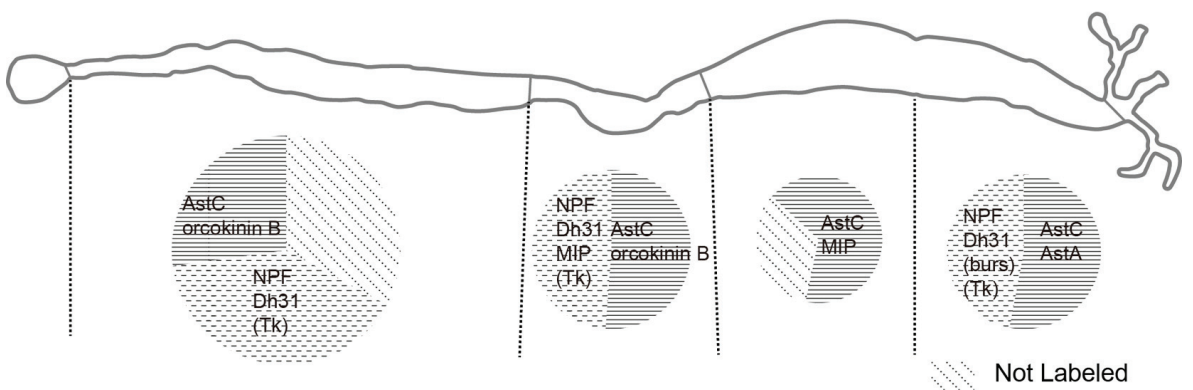

Fig. 7. Expression map of regulatory peptides that express in the Drosophila midgut. The midgut was divided into four regions, and the regulatory peptides expressed in each region are indicated. The sizes of the circle graphs and the slices showing subpopulations expressing specific combinations of regulatory peptides depict relative sizes based on the numbers in Table 1. the midgut, while additionally expressing NPF in the anterior and middle midgut, and Dh31 and Burs in the posterior midgut. The caudal half of the posterior midgut is a point of interest in the enteroendocrine peptide expression map that we constructed. Two subpopulations of enteroendocrine cells were defined in this region in previous studies (Beehler-Evans and Micchelli, 2015; Veenstra and Ida, 2014), and we added the expression of Dh31 and Burs to construct a more comprehensive map. In Drosophila larvae, midgut contractions are stimulated by tachykinin and Dh31 (LaJeunesse et al., 2010; Vanderveken and O'Donnell, 2014) and inhibited by AstA (Vanderveken and O'Donnell, 2014). Although regulatory peptide expression in the larval and adult midgut are not identical (Veenstra, 2009; Veenstra et al., 2008), it is likely that physiology such as digestion or feeding is regulated depending on the balance between enteroendocrine cells expressing specific peptides. In addition, only enteroendocrine cells that express both tachykinin and Dh31 are activated by protein and amino acids (Park et al., 2016), providing evidence that enteroendocrine cell populations expressing specific combinations of peptides have different functional properties. In our expression map, some regulatory peptides that express in the anterior midgut and anterior part of the posterior midgut are omitted (Fig. 7), due to technical difficulties with co-localization experiments with other peptides. For example, $\mathrm{CCHa} 2$ should be included in the anterior midgut, since our in situ results show $\mathrm{CCHa} 2$ expression in the anterior midgut (Fig. 1A), and previous studies report that $\mathrm{CCHa2}$-expressing cells are independent from AstC- or tachykinin-expressing enteroendocrine cells ( $\mathrm{Li}$ et al., 2013; Veenstra and Ida, 2014). In the anterior part of the posterior midgut, roughly 50 Prospero-positive cells do not appear to express any regulatory peptide tested for expression in this and other studies.

We identify the regulatory peptide genes expressed in enteroendocrine cells of the Drosophila midgut using RT-PCR, in situ hybridization, antisera, and GAL4 drivers, and define subgroups of enteroendocrine cells that express these genes in specific combinations. Very little is known regarding the functions of regulatory peptides that express in enteroendocrine cells. In addition, the mechanisms by which enteroendocrine cells decide which peptides to express is as yet unknown. Studies of these mechanisms should lead to insight of the processes in how subpopulations of enteroendocrine cells necessary depending on physiological conditions are determined, and how necessary changes or maintenance of subpopulations are implemented in a constantly renewing enteroendocrine cell population. This study provides a co-localization map and reagents that should prove useful in these future studies.
Note: Supplementary information is available on the Molecules and Cells website (www.molcells.org).

\section{ACKNOWLEDGMENTS}

We would like to thank Drs. Young-Joon Kim (GIST, Republic of Korea), Jan. A. Veenstra (Universite de Bordeaux, France), Benjamin White (NIH/NIMH), Mark R. Brown (University of Georgia), Developmental Studies Hybridoma Bank, Drosophila Genomics Resource Center, and the Bloomington stock center for reagents. This work was supported by the National Research Foundation of Korea (NRF) grant funded by the Korean Government (NRF-2013R1A1A2061120).

\section{REFERENCES}

Beehler-Evans, R., and Micchelli, C.A. (2015). Generation of enteroendocrine cell diversity in midgut stem cell lineages. Development 142, 654-664.

Brogiolo, W., Stocker, H., Ikeya, T., Rintelen, F., Fernandez, R., and Hafen, E. (2001). An evolutionarily conserved function of the Drosophila insulin receptor and insulin-like peptides in growth control. Curr. Biol. 11, 213-221.

Brown, M.R., Crim, J.W., Arata, R.C., Cai, H.N., Chun, C., and Shen, P. (1999). Identification of a Drosophila brain-gut peptide related to the neuropeptide $Y$ family. Peptides 20, 1035-1042.

Chen, Y., Veenstra, J.A., Davis, N.T., and Hagedorn, H.H. (1994). comparative study of leucokinin-immunoreactive neurons in insects. Cell Tissue Res. 276, 69-83.

Chen, J., Choi, M.S., Mizoguchi, A., Veenstra, J.A., Kang, K., Kim, Y.J., and Kwon, J.Y. (2015). Isoform-specific expression of the neuropeptide orcokinin in Drosophila melanogaster. Peptides 68, 50-57.

Dubreuil, R.R. (2004). Copper cells and stomach acid secretion in the Drosophila midgut. Int. J. Biochem. Cell Biol. 36, 745-752.

Engelstoft, M.S., Egerod, K.L., Lund, M.L., and Schwartz, T.W. (2013). Enteroendocrine cell types revisited. Curr. Opin. Pharmacol. 13, 912-921.

Hansen, K.K., Hauser, F., Williamson, M., Weber, S.B., and Grimmelikhuijzen, C.J. (2011). The Drosophila genes CG14593 and CG30106 code for G-protein-coupled receptors specifically activated by the neuropeptides CCHamide-1 and CCHamide-2. Biochem. Biophys. Res. Commun. 404, 184-189.

Hergarden, A.C., Tayler, T.D., and Anderson, D.J. (2012). Allatostatin-A neurons inhibit feeding behavior in adult Drosophila. Proc. Natl. Acad. Sc.i USA 109, 3967-3972.

LaJeunesse, D.R., Johnson, B., Presnell, J.S., Catignas, K.K., and Zapotoczny, G. (2010). Peristalsis in the junction region of the Drosophila larval midgut is modulated by DH31 expressing enteroendocrine cells. BMC Physiol. 10, 14

Lee, T., and Luo, L. (1999). Mosaic analysis with a repressible cell marker for studies of gene function in neuronal morphogenesis. Neuron 22, 451-461.

Lee, G., and Park, J.H. (2004). Hemolymph sugar homeostasis and starvation-induced hyperactivity affected by genetic manipulations of the adipokinetic hormone-encoding gene in Drosophila 
melanogaster. Genetics 167, 311-323.

Lee, K.S., You, K.H., Choo, J.K., Han, Y.M., and Yu, K. (2004). Drosophila short neuropeptide $F$ regulates food intake and body size. J. Biol. Chem. 279, 50781-50789.

Lee, K.S., Kwon, O.Y., Lee, J.H., Kwon, K., Min, K.J., Jung, S.A., Kim, A.K., You, K.H., Tatar, M., and Yu, K. (2008). Drosophila short neuropeptide $F$ signalling regulates growth by ERKmediated insulin signalling. Nat. Cell Biol. 10, 468-475.

Li, S., Torre-Muruzabal, T., Sogaard, K.C., Ren, G.R., Hauser, F., Engelsen, S.M., Podenphanth, M.D., Desjardins, A., and Grimmelikhuijzen, C.J. (2013). Expression patterns of the Drosophila neuropeptide CCHamide-2 and its receptor may suggest hormonal signaling from the gut to the brain. PLoS ONE 8, e76131.

Luan, H., Lemon, W.C., Peabody, N.C., Pohl, J.B., Zelensky, P.K., Wang, D., Nitabach, M.N., Holmes, T.C., and White, B.H. (2006). Functional dissection of a neuronal network required for cuticle tanning and wing expansion in Drosophila. J. Neurosci. 26, 573584.

Melcher, C., and Pankratz, M.J. (2005). Candidate gustatory interneurons modulating feeding behavior in the Drosophila brain. PLoS Biol. 3, e305.

Micchelli, C.A., and Perrimon, N. (2006). Evidence that stem cells reside in the adult Drosophila midgut epithelium. Nature 439, 475-479.

Min, S., Chae, B., Jang, Y.H., Choi, S., Lee, S., Jeong, Y.T., Jones, W.D., Moon, S.J., Kim, Y.J., and Chung, J. (2016). Identification of a peptidergic pathway critical to satiety responses in Drosophila. Curr. Biol., in press.

Nassel, D.R., and Winther, A.M. (2010). Drosophila neuropeptides in regulation of physiology and behavior. Prog. Neurobiol. 92, 42104.

Ohlstein, B., and Spradling, A. (2006). The adult Drosophila posterior midgut is maintained by pluripotent stem cells. Nature 439 , 470-474.

Park, J.H., and Kwon, J.Y. (2011). A systematic analysis of Drosophila gustatory receptor gene expression in abdominal neurons which project to the central nervous system. Mol. Cells 32, 375-381.

Park, S., Sonn J.Y., Oh Y., Lim C., and Choe J. (2014). SIFamide and SIFamide receptor defines a novel neuropeptide signaling to promote sleep in Drosophila. Mol. Cells 37, 295-301.

Park, J.H., Chen, J., Jang, S., Ahn, T.J., Kang, K., Choi, M.S., and Kwon, J.Y. (2016). A subset of enteroendocrine cells is activated by amino acids in the Drosophila midgut. FEBS Lett., in press.

Price, M.D., Merte, J., Nichols, R., Koladich, P.M., Tobe, S.S., and Bendena, W.G. (2002). Drosophila melanogaster flatline encodes a myotropin orthologue to Manduca sexta allatostatin. Peptides 23, 787-794.
Psichas, A., Reimann, F., and Gribble, F.M. (2015). Gut chemosensing mechanisms. J. Clin. Invest. 125, 908-917.

Reiher, W., Shirras, C., Kahnt, J., Baumeister, S., Isaac, R.E., and Wegener, C. (2011). Peptidomics and peptide hormone processing in the Drosophila midgut. J. Proteome Res. 10, 18811892.

Scopelliti, A., Cordero, J.B., Diao, F., Strathdee, K., White, B.H. Sansom, O.J., and Vidal, M. (2014). Local control of intestinal stem cell homeostasis by enteroendocrine cells in the adult Drosophila midgut. Curr. Biol. 24, 1199-1211.

Siviter, R.J., Coast, G.M., Winther, A.M., Nachman, R.J., Taylor, C.A., Shirras, A.D., Coates, D., Isaac, R.E., and Nassel, D.R. (2000). Expression and functional characterization of a Drosophila neuropeptide precursor with homology to mammalian preprotachykinin A. J. Biol. Chem. 275, 23273-23280.

Song, W., Veenstra, J.A., and Perrimon, N. (2014). Control of lipid metabolism by tachykinin in Drosophila. Cell Rep. 9, 40-47.

Vanderveken, M., and O'Donnell, M.J. (2014). Effects of diuretic hormone 31, drosokinin, and allatostatin A on transepithelial $\mathrm{K}(+)$ transport and contraction frequency in the midgut and hindgut of larval Drosophila melanogaster. Arch. Insect Biochem. Physiol. 85, 76-93.

Veenstra, J.A. (2009). Peptidergic paracrine and endocrine cells in the midgut of the fruit fly maggot. Cell Tissue Res. 336, 309-323.

Veenstra, J.A., and Ida, T. (2014). More Drosophila enteroendocrine peptides: Orcokinin $\mathrm{B}$ and the CCHamides 1 and 2. Cell Tissue Res. 357, 607-621.

Veenstra, J.A., Agricola, H.J., and Sellami, A. (2008). Regulatory peptides in fruit fly midgut. Cell Tissue Res. 334, 499-516.

Wang, C., Guo, X., Dou, K., Chen, H., and Xi, R. (2015). Ttk69 acts as a master repressor of enteroendocrine cell specification in Drosophila intestinal stem cell lineages. Development 142, 33213331.

Wegener, C., and Veenstra, J.A. (2015). Chemical identity, function and regulation of enteroendocrine peptides in insects. Curr. Opin. Insect Sci. 11, 8-13.

Williamson, M., Lenz, C., Winther, A.M., Nassel, D.R., and Grimmelikhuijzen, C.J. (2001). Molecular cloning, genomic organization, and expression of a B-type (cricket-type) allatostatin preprohormone from Drosophila melanogaster. Biochem. Biophys. Res. Commun. 281, 544-550.

Wu, Q., Wen, T., Lee, G., Park, J.H., Cai, H.N., and Shen, P. (2003) Developmental control of foraging and social behavior by the Drosophila neuropeptide Y-like system. Neuron 39, 147-161.

Zeng, X., and Hou, S.X. (2015). Enteroendocrine cells are generated from stem cells through a distinct progenitor in the adult Drosophila posterior midgut. Development 142, 644-653. 